\title{
A VEHICLE - BRIDGE INTERACTION
}

The problem of a vehicle - bridge interaction can be followed in the literature since the year 1849. At the early state the analytical methods were applied. The development of computers brings the change in the approach to the used methods of solution. The Finite Element Method and Component Element Method represent the revolution and qualitative jump in the development. They enable to solve very truthful space computing models numerically. The results of numerical computation must by verified by the in situ experimental measurements. Mutual interconnection of numerical and experimental techniques represents the most effective way in the process of uncovering of objective reality concerning of real action of bridge structure under the passing of vehicles.

\section{Introduction}

The solution of problems of a vehicle - bridge interaction belongs to the oldest solved problems of structural dynamics. The works of the civil engineer Willis [1] and mathematician Stokes [2] in which they tried to clarify the breakdown of Chester Rail Bridge in England in 1847 are not only considered the first attempts to solve the problems of a vehicle - bridge interaction but also the first works in the field of structural dynamics. The works of Krilov [3] and Timosenko [4] concerning mass less force movement along mass beam started a series of approximate solutions of the problem with various types of boundary conditions and load. Inglis monograph [5] brings a survey of these early studies. A more serious attention to the dynamic of highway bridges starts to be paid in $20^{\text {th }}$ century. The $1 \mathrm{st}$ important report on this topic was published in 1931 by the Special Committee on Highway Bridges of the American Society of Civil Engineers [6]. The year 1950 can be considered a fault in the development by the use of digital and analog computers and measuring technique. A detailed and systematic research into the vehicle - bridge interaction problems was carried out at the University of Illinois, Urbana, USA from 1950 to 1960 The results of this research were published by the Committee on Deflections Limitations of Bridges of the Structural Division of the ASCE in [7]. The complete survey of advances used till 1959 was published by Wright and Green in [8]. The total review of the world development till 1975 of the vehicle - highway bridge interaction problems was published by Tseng Huang in [9]. This period is characterized by using the plane computing models of vehicles and bridges with a finite number of degrees of freedom. Equations of motion are derived in the form of differential equations and are solved numerically by some step-by-step integration methods. Revolution in the solution of vehicle - bridge interaction problems represents the invasion of finite methods especially the Finite Element Method and Finite Strip Methods [10]. This enables to analyse the space computing models of bridges and vehicles. Computing models of vehicles are usually created by the use of the Component Element Methods [11]. Development of statistical methods initiated the modelling of surface unevenness by power spectral densities of unevenness and application of numerical simulation methods in the modelling of fatigue processes and lifetime of structures. The Monte Carlo Method is currently used at the modelling of stochastic processes. Possibilities of personal computers and software in the area of user environment orientate the progress to assurance the maximal user comfort and obtaining the effective graphical outputs. Display of graphical outputs is connected with picture animation.

Bridge engineering in the Slovak and Czech Republic has rich tradition, high technical level and positive response all over the world. The works of professor Koloušek in the theoretical and experimental fields laid the foundation of a new branch of science - Dynamic of Structure an especially Dynamic of Bridges in our country [12]. Problems of a vehicle - railway bridge interactions are solved in the Railway Research Institute (VÚŽ) and in the Institute of Theoretical and Applied Mechanics of the Czech Academy of Sciences (ÚTAM AV ČR) in Prague. Research in the field of a vehicle - highway bridge interaction was formed step by step following the road network infrastructure construction. The Department of Structural Mechanics of the University of Zilina and the Department of Structural Mechanics of the Czech Technical University in Prague contributed substantially to the mentioned research. Besides theoretical research the systematic attention was paid to the development of experimental methods on the Civil Engineering Research Institute (VÚIS), the Institute of Construction and Architecture of the Slovak Academy of Sciences in Bratislava (ÚSTARCH SAV) and the Building Testing and Research Institute in Bratislava and Prague (TSÚS). The problems of vehicle - bridge interactions are solved in monographs [13], [14]. The present development aims at the solution of specific problems, e.g. optimal control of bridge - friendly vehicle [15].

\footnotetext{
* Jozef Melcer

Department of Structural Mechanics, Faculty of Civil Engineering, University of Žilina, Slovakia, E-mail: melcer@fstav.utc.sk
} 


\section{Description of the problem}

Real admission to the solution of the problem of a vehicle bridge interaction comes out from the mutual combination of numerical and experimental approaches. Numerical methods in the present days offer an effective tool for the solution of this problem. If the entering values are put into calculation in regular magnitudes and verified on the basis of experimental measurements, the results of numerical calculations correspond to the results obtained experimentally. The contemporary state of computing technology enables to solve all the problems in real time. For the creation of computing programs it is suitable to use the high-level program languages. The results obtained from the numerical and experimental analyses are used in the process of construction of transport means and in the design of optimal parameters of bridges with respect to its lifetime and reliability. In the relation to the passengers the results pay attention to the ride comfort of passengers.

Numerical solution of the problem of a vehicle - bridge interaction requires to consider at least the following issues:

- creation of a vehicle computing model and its mathematical description,

- creation of a bridge computing model and its mathematical description,

- modelling and mathematical description of the surface unevenness,

- creation of a computer program for numerical solution of equations of motion,

- creation of a computer program for evaluation and display of the obtained data.

There are many concrete techniques how to create computing models of vehicles and bridges. Basically, they can be divided into two categories on the basis of a resulting type of equations of motion. Equations of motion can be formed as differential or integral equations. The possibilities of numerical solution of both types of equations of motion are in the present time very large. It is practically the question of individual approach of the author.

Computing models of vehicles are usually created as discrete models composed of systems of mass points or mass bodies, discrete springs and dampers, or they are created in the sense of Finite Element Methods. Such computing models are described by a system of ordinary differential equations. In the Department of Structural Mechanics, Faculty of Civil Engineering in Zilina the Component Element Methods is successfully used for creation of computing models of vehicles [11]. In compliance of this Method the equations of motion can be written in the form

$$
\left[m_{v}\right] \cdot\{\ddot{r}(t)\}=\left\{F_{v}(t)\right\},
$$

where $\left[m_{v}\right]$ is the mass matrix of vehicle, $\{\ddot{r}(t)\}$ is the vector of second derivatives of displacement components corresponding to individual degrees of freedom and $\left\{F_{v}(t)\right\}$ vector is the complete vector of forces acting on a vehicle. The sample of $3 \mathrm{D}$ computing models of vehicle is plot in Fig. 1 .

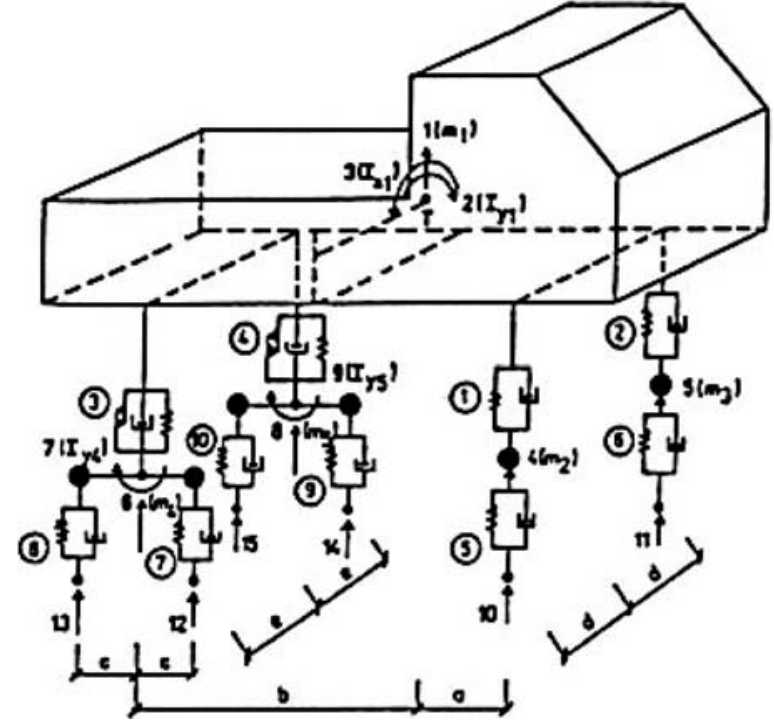

Fig. 13 computing model of vehicle.

The bridge structure can be modelled as a system with continuously distributed mass or as a system with a finite degree of freedoms. Nowadays, bridge structures are modelled in compliance with FEM where the computing model is created as an arbitrary combination of line 1D elements, slab - wall or shell 2D elements or $3 \mathrm{D}$ space elements. The equations of motion in a matrix form described in compliance with FEM forced vibration of a bridge can be written as

$$
[M] \cdot[\ddot{u}]+[B] \cdot[\dot{u}]+[K] \cdot[u]=\{F(t)\} .
$$

$[M]$ is the global mass matrix, $[B]$ is the global damping matrix, $[K]$ is the global stiffness matrix, $\{u\}$ is the global vector of unknown nodal parameters of displacements (vector of generalized nodal displacements) and $\{F(t)\}$ is the vector of excitation forces transformed into nodal points in compliance with FEM. In Fig. 2 the FEM computing model of two span bridge structure is showen.

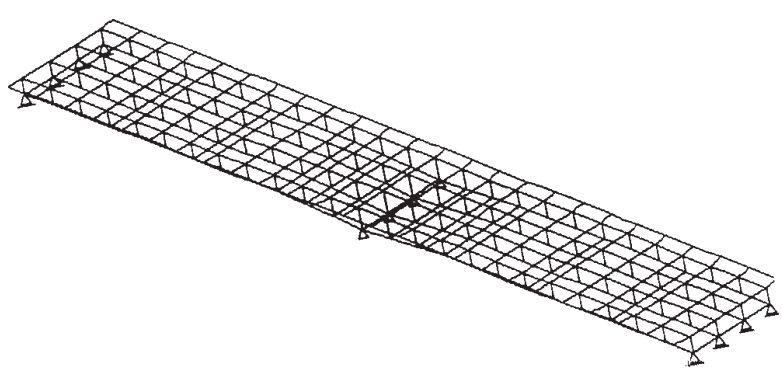

Fig. 2 Two span FEM computing model of a bridge.

A mathematical description of the road surface unevenness is also an integral part of the problem. The road surface unevenness can be defined as deterministic or stochastic. The local discrete 
unevenness and periodically repeated unevenness are modeled as deterministic. A terrain step represents a specific case of deterministic unevenness. From a mathematical point of view it is a function with point singularity. For the purpose of numerical modeling of such unevenness it is better to substitute the real shape of the terrain step by an alternate function identical with the function describing the trajectory of the vehicle wheel hub. At present the randomly variable road profile is derived from power spectral densities of unevenness for a certain category or pavement and the quality of its surface on the basis of in situ experimental measurements in the form [16].

$$
S(\Omega)=C . \Omega^{-2},
$$

where $C$ [rad.m] is a parameter describing the measure of unevenness and $\Omega$ is so called length angular frequency in $\left[\mathrm{rad} . \mathrm{m}^{-1}\right.$ ]. When the coordinates on the vertical and horizontal axes are plotted in a logarithmical measure, the power spectral densities of unevenness can be very well approximated by a straight line, Fig. 3 .

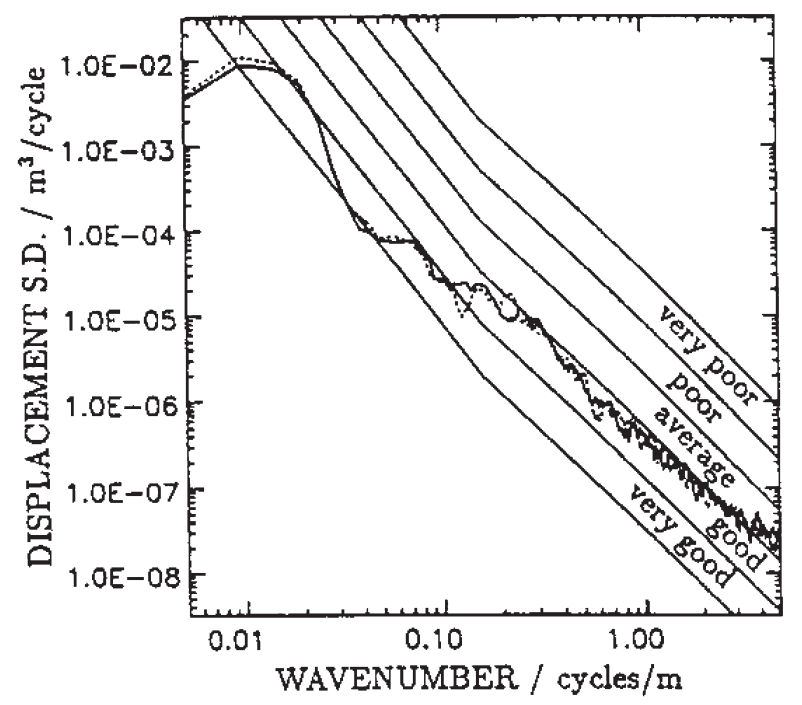

Fig. 3 Power spectral densities of road unevenness according to [16]

Equations of motion describing the vehicle and bridge vibration are solved numerically. For the solution a numerical integration method is applied. The whole calculation is realized in time domain with time step $\Delta t$. From surface unevenness and from bridge displacements the displacements of contact points of vehicle are determined. From equations of motions describing the vehicle vibration the contact forces corresponding to such kinematical excitation are calculated. The bridge response is calculated on the contact forces representing the excitation forces inducing the bridge vibration. After the calculation of the bridge response the vehicle is moved forward about the length corresponding to the time step $\Delta t$ and the whole cycle is repeated.

In the Department of Structural Mechanics, Faculty of Civil Engineering in Zilina the equations of motion are usually com- piled as differential equation and they are solved numerically. Various methods are used for numerical solution of differential equations. For example, the central difference method, Newmark's method, Wilson's method, predictor-corrector method. We have very good experience is with the use of Runge-Kutta $4^{\text {th }}$ or $5^{\text {th }}$ order method.

In the present time it is optimal to use a higher level programming language, for example MATLAB, to create a computing program. This programming language is matrix oriented, interactive and computing procedures are supported by effective graphics. The creation of a program is easier in comparison with classical program languages. For example, the program statement for a numerical integration of differential equations by the Runge-Kutta $4^{\text {th }}$ order integration method can be written in one line

$$
[t, y]=o d e 45(\text { 'vozidlo', }[t 0, t \text { final }],[\text { InKon }]) .
$$

The symbols in the former statement have the following meaning: ode 45 is a matlab function for integration of differential equations by the Runge-Kutta $4^{\text {th }}$ order integration method. In the parentheses behind this function in apostrophes is the name of functional file in which the system of solving equations is stored 'vozidlo', in the 1st brackets [ $\mathrm{t} 0$, tfinal] contain the initial and final values of the time interval in which the numerical integration is carried out and in the $2^{\text {nd }}$ brackets [InKon] contains initial conditions (initial displacements and initial speeds of integrated functions). This statement recalls vector $t$ time steps of numerical integration and column matrix $y$ in which the functional values of wanted functions are stored.

In the past two program systems for analysis of the problems of a vehicle - bridge interaction and for the processing of obtained results were created. The $1^{\text {st }}$ program system BRIDGEW2 was created in the program language FORTRAN. In this program the Runge-Kutta $5^{\text {th }}$ order integration procedure was adopted. Some working windows of the program system are presented in Fig. 4 . The $2^{\text {nd }}$ program system ISV was created in language $\mathrm{C}++$ and for integration of differential equations the Central Difference Method was employed.

\section{Application in practice}

The Old Bridge in Bratislava was erected in the year 1945 as a temporary bridge of the Roth-Wanger system, but it serves for transport needs up to these days, Fig. 5. The $1^{\text {st }}$ bridge span from Bratislava water side $75 \mathrm{~m}$ long acts as a single beam. This span has been strengthened and statically and dynamically tested many times. The simulation of a vehicle motion along the bridge span was carried out for the need of tuned vibration control of the bridge. Some comparison of the numerically and experimentally obtained results, corresponding to the speed of vehicle motion $42 \mathrm{~km} / \mathrm{h}$, is presented in the following text.

The planar computing model of a vehicle and bridge was adopted for numerical analysis. Equations of motions were derived in 

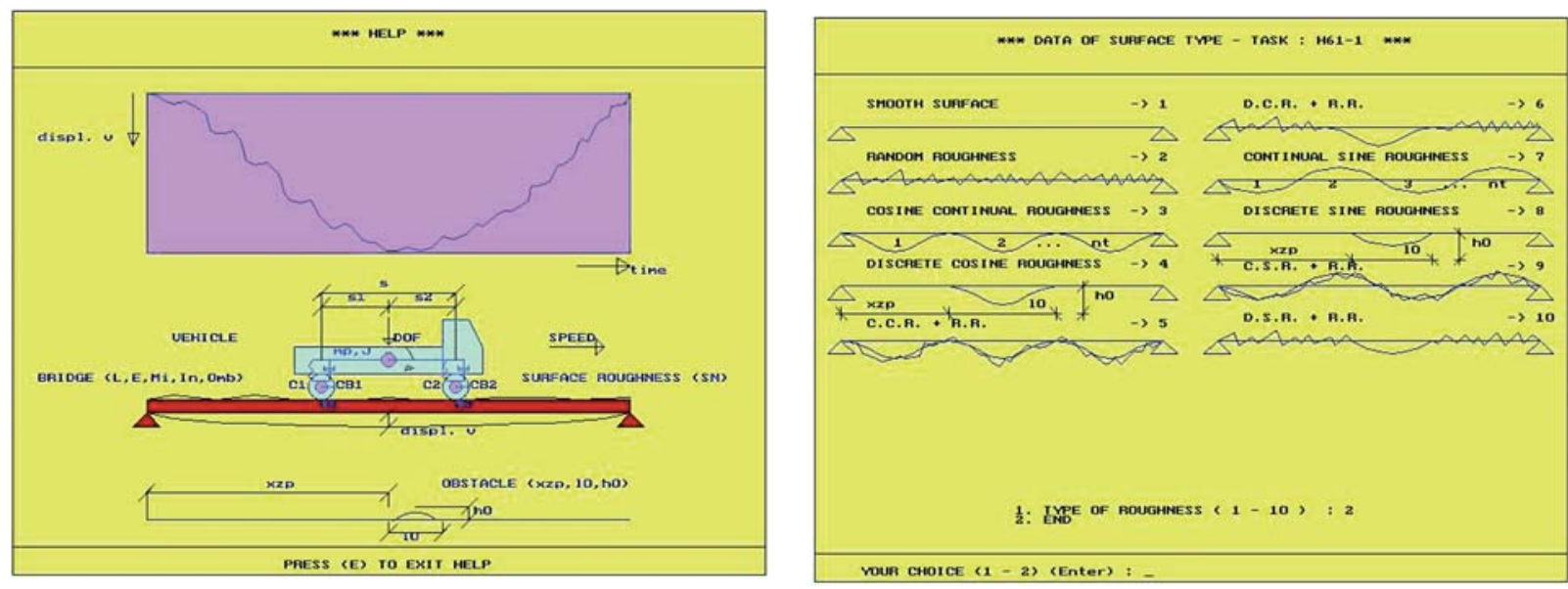

Fig. 4 Some working views of program system BRIDGEW2 according to [14]

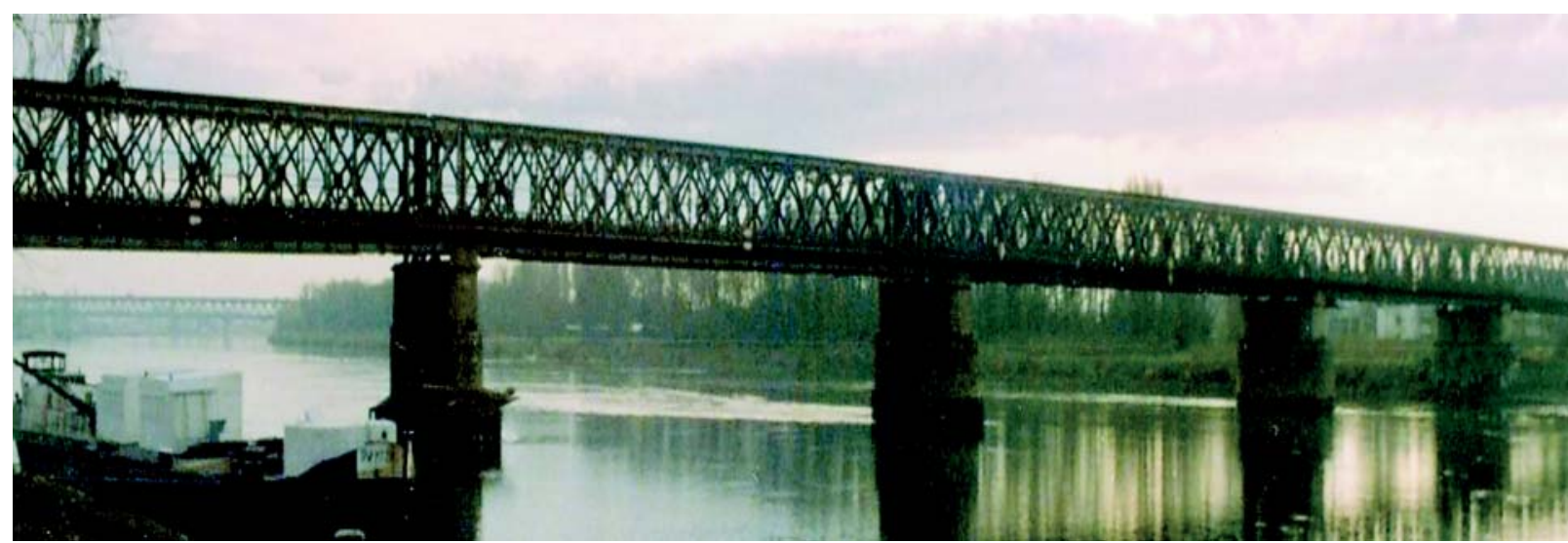

Fig. 5 Old Bridge in Bratislava

[mm]

b/4. 7

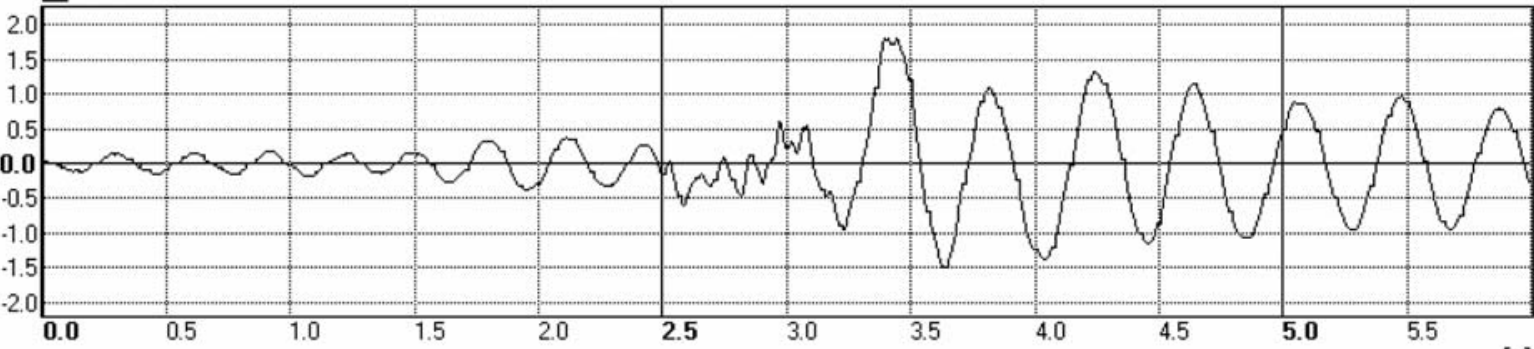

Fig. 6 Experimentally obtained time record of vertical mid span deflection

the form of differential equations and were solved numerically with the help of Runge-Kutta $5^{\text {th }}$ order integration procedure. The vertical dynamic mid span deflections were numerically and experimentally tested under the vehicle passing over a standard obstacle. The results were mutually compared, Fig, 6 and Fig. 7.
As we can see the mutual agreement between the experimentally and numerically obtained results is very good, especially in the part of the record corresponding to the driving of the vehicle behind the obstacle. A very good agreement is also at a numerically modelled frequency composition of vibration and at experi- 


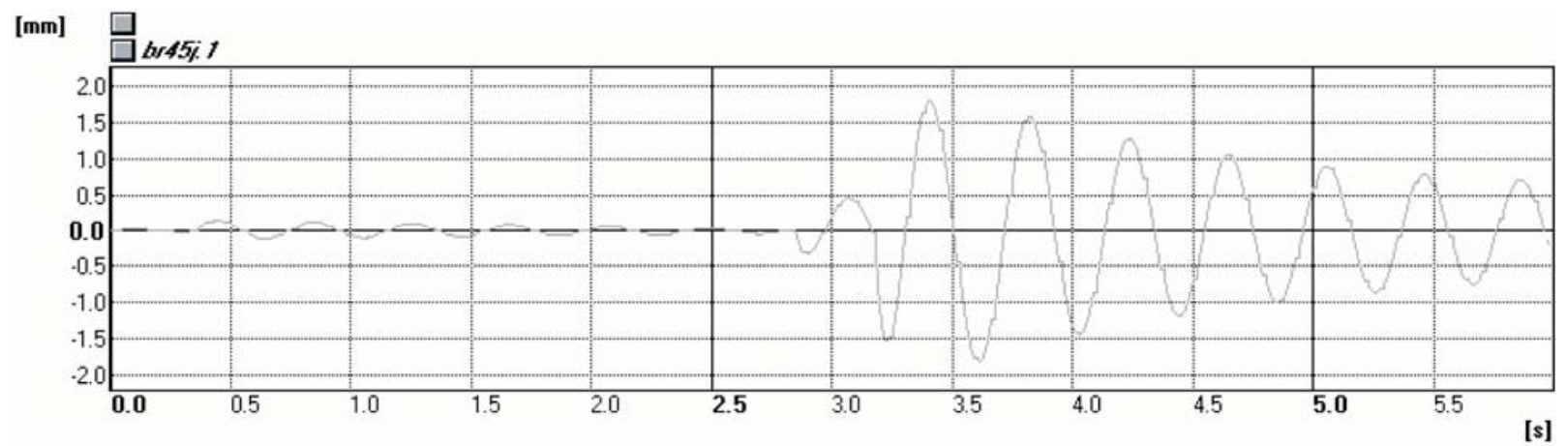

Fig. 7 Numerically obtained time record of vertical mid span deflection

mentally obtained frequency composition of vibration. It is seen very well from the power spectral density functions (PSDF), Fig. 8 and Fig. 9.

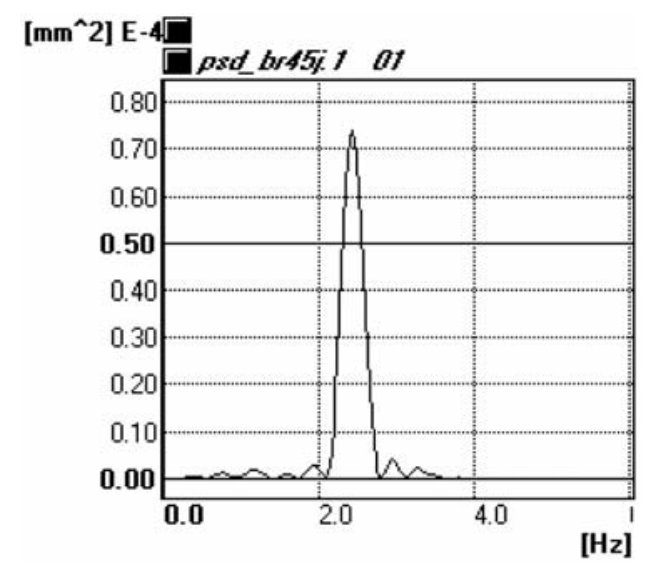

Fig. 8 Experimentally obtained PSDF

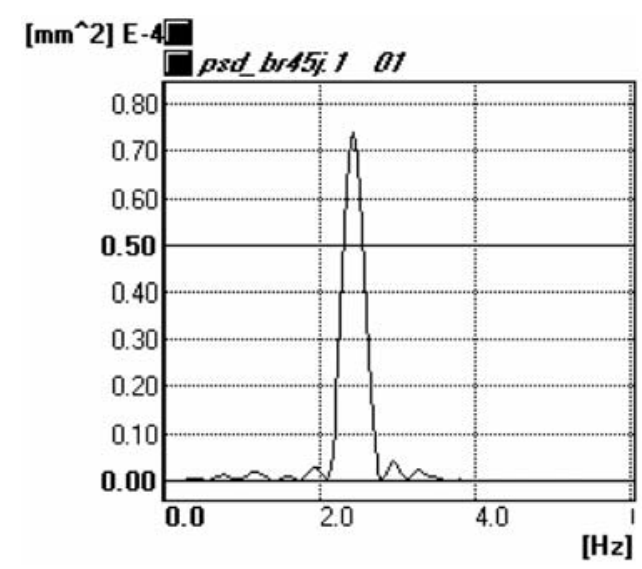

Fig. 9 Numerically obtained PSDF
The coherence function, Fig. 10, confirms practically $100 \%$ agreement of the experimentally and numerically obtained vibration records in the frequency range from 2 to $3 \mathrm{~Hz}$, which is the area of maximal power.

I

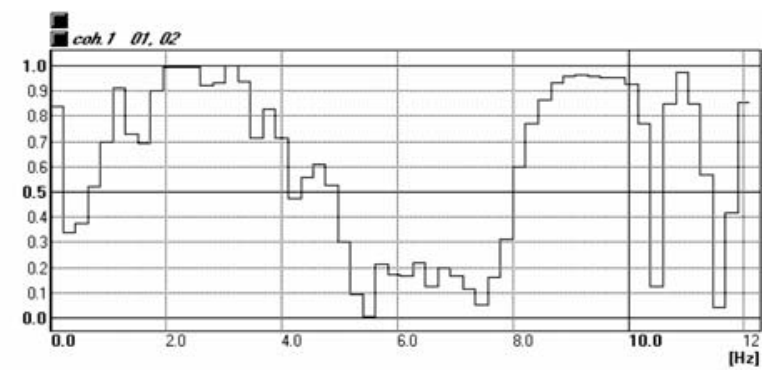

Fig. 10 Coherence function for experimentally and numerically obtained vibration records

\section{Conclusions}

The vehicle - bridge interaction problem represents the oldest problem of structural dynamic at all. Contemporary approach to the solution of this problem comes out from a mutual combination of numerical and experimental approaches. Numerical methods offer an effective tool for the solution of the problem in the present days. The contemporary state of computing technology enables to solve all the problems in real time. It is suitable to use the highlevel program languages for the creation of computing programs. The results obtained from the numerical and experimental analyses are used for the design of optimal bridge parameters and for specification of lifetime and reliability recommendations. 


\section{References}

[1] WILLIS, R.: Appendix, Report of the Commissioners Appointed to Inquire into the Application of Iron to Railway Structures, Stationary Office, London, 1849.

[2] STOKES, G. G.: Discussion of a Differential Equation Relating to the Breaking of Railway Bridges, Transactions Cambridge Philosophic Society, 8, 1849, p. 707.

[3] KRYLOV, A. N.: Über die erzwungenen Schwingungen von gleichförmigen elastischen Stäben, Mathematische Annalen, Jahrg.61, 1905, s.221. (From: Matematičeskij zbornik Akademii Nauk, Vyp. 61, Peterburg, 1905.)

[4] TIMOŠENKO, S. P.: Forced vibration of prismatic bars (in Russian), Izvestija Kievskogo politechničeskogo instituta, 1908.

[5] INGLIS, C. E.: A Mathematical Treatise on Vibrations in Railway Bridges, Cambridge University Press, Cambridge, Mass., 1934.

[6] Impact on Highway Bridges, Final Report of the Special Committee on Highway Bridges, Transactions ASCE, Vol. 95, 1931, p. 1089-1117.

[7] Deflection Limitations of Bridges, Progress Report of the Committee on Deflection Limitations of Bridges of the Structural Division, Proc. ASCE, Vol. 84, No. ST3, May, 1958, p. 1633.

[8] WRIGHT, D. T., - GREEN, R.: Highway Bridge Vibration, Review of Previous Studies. Queen s University Kingston, Ontario, 1959.

[9] HUANG, T.: Vibration of Bridges. Shock and Vibration Digest, 3/1976, p. 61-76.

[10] BATHE, K. J.: Finite Element Procedures in Engineering Analysis, Prentice-Hall, New Jersey, 1982.

[11] LEVY, S., WILKINSON, J. P. D.: The Component Element Method in Dynamics with Application to Earthquake and Vehicle Engineering, McGraw-Hill, New York, 1976.

[12] KOLOUŠEK, V.: Dynamics of Structures I, II, III. (in Czech), SNTL, Prague, 1954, 1956, 1961.

[13] FRÝBA, L.: Dynamic of Railway Bridges (in Czech), ACADEMIA, Prague, 1992.

[14] MELCER, J.: Dynamic Calculation of Highway Bridges (in Slovak), EDIS, University of Zilina, 1997.

[15] MÁCA, J., ŠMILAUER, V., VALÁŠEK, M.: Optimal Control of Bridge - Friendly Trucks, Proc. III International Conference on New Trends in Statics and Dynamics of Buildings, Slovak republic, Bratislava, Civil Engineering Faculty STU, October 21 - 22, 2004, STU Bratislava, p. 109 - 112.

[16] CEBON, D.: Handbook of vehicle - road interaction, Swets\&Zeitlinger B.V. Publishers, Lisse, Netherlands, 1999. 